\title{
EL VIRAJE EPISTEMOLÓGICO DE WERNER HEISENBERG
}

\author{
Josefa Castellà Cid \\ Tarragona
}

\begin{abstract}
Resumen: El objetivo de este trabajo es estudiar la evolución de la perspectiva epistemológica que experimentó Heisenberg, evolución paralela a la que también experimentó Einstein. Así, Heisenberg fue influido por el segundo, en un principio como creador de la teoría especial de la relatividad y posteriormente como creador de la teoría general de la relatividad, y ambos, en sus inicios, fueron inspirados por los lemas del positivismo de Mach, aunque después se alejaron de esta corriente de pensamiento. Este viraje se presenta paralelo al giro que ambos físicos también realizaron en sus respectivos dominios de investigación y muestra la tensión que sufre el científico que pretende comprender el mundo y se encuentra ante el siguiente dilema: limitarse a los hechos observables o distanciarse para ver las relaciones formales que se dan entre ellos.
\end{abstract}

Palabras clave: Mecánica cuántica, positivismo, observable, relación formal.

\section{The Epistemological Variation of Werner Heisenberg}

Abstract: This paper focuses on the development of the Heisenberg's epistemological perspective, which ran parallel to Einstein's. Therefore, we can say that Heisenberg was influenced by Einstein, as the creator of the special and general theories of relativity and relativity. Both scientists were inspired in their start by the mottos of Mach's positivism but afterwards they got away from this line of thought. This change was in tune with their change in their respective research fields, and shows the tension suffered by the scientist who seeks to understand the world and is faced with the following dilemna: either limit oneself to observable facts or get away from them in order to see the formal relationships among them.

Keywords: Quantum Mechanics, positivism, observable, formal relationship.

Recibido: 14/11/2016 Aprobado: 21/4/2017 
Josefa Castellà

\section{Introducción}

El desarrollo de la moderna mecánica cuántica se inició en el verano de 1925, cuando Heisenberg (1901-1976) concibió la idea de representar las cantidades físicas en conjuntos de números complejos y creó así la mecánica de matrices, que constituyó la primera formulación matemática de la teoría cuántica. A principios de 1927, Heisenberg derivó las relaciones de indeterminación, que son uno de los elementos fundamentales de la interpretación de Copenhague, la interpretación del formalismo cuántico que mayor aceptación ganó entre los físicos.

Tras estas aportaciones a la fundamentación de la mecánica cuántica había un fondo conceptual, que nos lleva a preguntar: ¿Qué presuposiciones epistemológicas las guiaron? ¿De qué preconcepciones surgieron? Los pensamientos de Heisenberg no surgieron de la nada, sino que fueron estimulados por los descubrimientos de la física atómica de la época, por las dificultades conceptuales que presentaban para ser encajados con las teorías físicas existentes, por las cuestiones metafísicas y epistemológicas que planteaban y por las apasionadas polémicas que provocaban.

Encontramos el carácter filosófico del discurso de Heisenberg en repetidas ocasiones, como en la revelación que hizo a M.Bunge:"Mi motivación e inspiración heurísticas han sido siempre de naturaleza filosófica" (Cassidy, 2002:2). Pronunció estas palabras cuando se quejaba de una generación de físicos teóricos — refiriéndose a los que consideraba de mentalidad positivista- que pretendían regresar a Ptolomeo: "Solo desean describir y predecir los hechos. En cambio, yo soy de mente newtoniana. Quiero entender los 
hechos". Para entender los hechos empíricos que observaba, Heisenberg desarrolló unos pensamientos que tuvieron gran repercusión en el campo de la física, de la filosofía y del humanismo en general. La mecánica de matrices es uno de los pilares de la mecánica cuántica y las relaciones de indeterminación son una de las claves de su interpretación. ¿Cuál fue la perspectiva epistemológica bajo la cual fueron desarrolladas? ¿Cómo evolucionó esta perspectiva?

Sin embargo, a pesar de que Heisenberg es, por una parte, uno de los arquitectos del formalismo de la mecánica cuántica y, por otra, es uno de los creadores de la interpretación filosófica predominante de dicho formalismo, cuarenta años después de su muerte, su pensamiento filosófico y su perspectiva epistemológica aún son poco conocidos. Dentro del pensamiento filosófico de Heisenberg, su aportación epistemológica presenta notable interés, porque constituye un conjunto de reflexiones que sobre la naturaleza de la ciencia física realizó un científico que dedicó toda su vida a la investigación física, consiguiendo grandes éxitos en su juventud —aunque no tantos en su madurez-. Esperamos mostrar el valor de dichas reflexiones en las páginas de este trabajo, dedicado a estudiar la perspectiva epistemológica de Heisenberg, especialmente su actitud ante el positivismo, corriente de pensamiento que dominaba el ambiente intelectual en el que inició su carrera e inspiró sus primeros y más significativos trabajos, los que le situaron en la cumbre de los creadores de la mecánica cuántica.

Heisenberg inició su histórico artículo de 1925, en el que presentaba su mecánica de matrices, exponiendo su objetivo de utilizar tan solo magnitudes observables. Al expresar su pretensión de utilizar solo observables estaba 
mostrando su perspectiva positivista. El artículo que al año siguiente publicó con Born y Jordan, en el que se desarrollaba la propuesta inicial de Heisenberg, también fue diseñado en un marco de pensamiento positivista, puesto que, ya en su introducción, mostraba el objetivo de sus autores de establecer un sistema matemático para la mecánica de los movimientos atómicos y electrónicos que fuera unitario y simple, como la mecánica clásica, pero que "consistiera enteramente en relaciones entre cantidades que son en principio observables".

Pero Heisenberg no siempre fue positivista. La conversación que, sobre los problemas de la mecánica cuántica, mantuvo con Einstein en 1926 empezó a sembrarle las dudas (Heisenberg, 1967: 88). Einstein le preguntó sobre el fundamento filosófico de la nueva teoría y le señaló que en esta no aparecía el concepto de "órbita de un electrón”. Heisenberg le respondió que esta órbita no se podía observar y, en cambio, las magnitudes de una teoría habían de ser directamente observables (Heisenberg, 1980: 122). Pero Einstein replicó que todas las teorías contienen magnitudes inobservables y solo la teoría decide lo que se puede observar. Y esta última proposición inspiró, meses después, el desarrollo de las relaciones de indeterminación (Heisenberg, 1967: 106). Sin embargo, este desarrollo, que aparece en el segundo artículo histórico de Heisenberg, el de 1927, aún contiene rasgos positivistas y operacionalistas. Su punto de partida es la concepción según la cual un concepto científico es un sistema de operaciones y su significado es una determinada relación de impresiones sensoriales del observador. Por ejemplo, para entender el significado del concepto "lugar" o "posición” de un electrón nos hemos de referir a un determinado procedimiento por el cual sea obser- 
vada la posición; si no es así el concepto "posición” se encuentra privado de significado ${ }^{1}$.

Heisenberg concluía su artículo de 1927 negando la ley de la causalidad, argumentando que si no se pueden determinar exactamente los valores iniciales no se pueden calcular tampoco los valores futuros. No es un argumento lógicamente correcto, porque la falsedad del antecedente no implica estrictamente la falsedad del condicional. Pero Heisenberg ya se había anticipado a esta objeción: desde su punto de vista positivista y operacionalista, inaplicabilidad e invalidez eran sinónimos. Según Heisenberg era posible preguntar si había oculto, tras el universo probabilístico que percibíamos, un mundo "verdadero" en el que la ley de la causalidad sería válida. Pero plantear esta cuestión le parecía inútil y sin sentido, puesto que la ciencia física solo debía describir la correlación de las observaciones.

Sin embargo, Heisenberg abandonó pronto esta actitud positivista. El viraje en su posición es una muestra del dilema ante el que se encuentra el científico, puesto que este pretende construir una ciencia segura y por esta razón, por una parte, piensa que ha de atenerse con rigor y suma cautela a los hechos observados, pero, por otra, busca entenderlos y, para ello, necesita realizar grandes abstracciones con las que encontrar las interconexiones que expliquen estos hechos. El giro en la posición epistemológica de Heisenberg se correspondió con el cambio que también realizó en su dominio de investigación, puesto que a medida que se dedicaba a investigar el campo de

${ }^{1} \mathrm{E} 1$ procedimiento puede consistir simplemente en iluminar el electrón y observarlo con el microscopio. 
materia unitario se iba alejando de los preceptos positivistas. Y el precepto que él mismo ofrecía a los jóvenes investigadores era que no hay que atarse a ningún pensamiento filosófico sino que hay seguir el que más contribuya al progreso de la ciencia física. Como vamos a ver, la duda sobre si la actitud positivista es o no es la más adecuada para el progreso de la ciencia natural se encuentra como trasfondo filosófico tras las contribuciones más significativas que Heisenberg realizó a la física, constituidas por su creación de la mecánica de matrices, su derivación de las relaciones de indeterminación y su propuesta de una teoría unificada de campo.

El presente trabajo se inicia con el estudio de la perspectiva epistemológica bajo la cual surgieron la mecánica de matrices y las relaciones de indeterminación. Era una perspectiva positivista, puesto que Heisenberg pretendía basar la mecánica cuántica solo en relaciones entre magnitudes observables. Pero esta perspectiva inicial experimentó una evolución, que le llevó a alejarse del ideario positivista y que se muestra también en sus consideraciones sobre la relación de causalidad.

Este trabajo estudia la influencia que Einstein ejerció en dicha metamorfosis. La evolución de la perspectiva epistemológica de Heisenberg, como la de Einstein, se relaciona con la evolución que experimentó en su ámbito de investigación. Einstein había sido inspirado por Mach al rechazar la existencia del espacio y el tiempo absolutos por no ser observables, pero al ampliar la teoría especial de la relatividad en la teoría general se había distanciado de los principios positivistas, puesto que le resultaban demasiado restrictivos para la construcción de una teoría cuyos enunciados generales estaban muy distantes del dominio de la directa observación empírica. Lo mismo le ocurrió a Hei- 
senberg cuando se introdujo en un nuevo dominio de investigación, la búsqueda de una teoría de un campo unificado que uniera todos los campos conocidos.

Los principios positivistas no habían ayudado a Heisenberg a encontrar la matriz de scattering. Y prescindió de ellos para encontrar el campo que unificara partículas y campos, el campo general de materia. Presentó una propuesta según la cual las cualidades de las partículas emergían de las propiedades de simetría de la ecuación de dicho campo general pero no consiguió su objetivo de encontrar una adecuada ecuación para este. Y mientras continuaba trabajando en esta búsqueda también intentó situar su trabajo en una perspectiva filosófica, que derivaba directamente de la antigua filosofía griega. Así, encontró en Platón la expresión de su idea motriz: la estructura fundamental de la materia está constituida por simetrías, formas matemáticas que determinan la disposición de las partículas elementales y muestran la simplicidad de los fenómenos y la unidad subyacente al mundo.

Comprender el mundo es captar formas simples que relacionan los diferentes fenómenos y es entender interconexiones que no son apreciables a través de la observación directa e inmediata de los sentidos. Comprender no consiste en pronosticar futuras observaciones, que es lo que según Heisenberg mantenían los positivistas. Por consiguiente, si se siguieran estrictamente los preceptos positivistas no se llegaría a comprender los fenómenos, que es el objetivo de la ciencia. Así pues, este trabajo concluye estudiando la actitud crítica que Heisenberg mantuvo en su madurez ante el ideario positivista que previamente le había inspirado en su creación de la mecánica de matrices. 
Josefa Castellà

\section{La mecánica de matrices}

\subsection{Contexto histórico}

A pesar del éxito que había alcanzado con los numerosos problemas de física atómica que había resuelto, la teoría cuántica, antes de 1925, no constituía una teoría lógicamente consistente sino que era un conjunto no sistematizado de hipótesis, principios, teoremas, reglas, y argumentos analógicos. El procedimiento que, en general, se seguía para solucionar un problema era el siguiente: en primer lugar se admitía que las partículas obedecían las leyes de la mecánica clásica y, en segundo lugar, se postulaba que de todas las soluciones obtenidas sólo se había de tener en cuenta las que satisfacían ciertas reglas de cuantificación establecidas ad hoc. Así, cada problema era solventado, en un principio, en términos de física clásica y, a continuación, la solución que se había obtenido se adaptaba, según el principio de correspondencia ${ }^{2}$, al lenguaje cuántico (Messiah, 1983: 31). Este proceso de adaptación exigía más intuición y habilidad que sistemática argumentación deductiva. Así se puede decir que a la teoría cuántica de aquella época le faltaban dos de las cualidades esenciales que ha de tener una teoría científica, la autonomía conceptual y la consistencia lógica (Jammer, 1989: 208).

${ }^{2}$ Por el cual, para números cuánticos elevados, los resultados inferidos por la teoría cuántica coinciden con los resultados derivados de las teorías clásicas. 
Esta era la situación con la que se encontró el joven Heisenberg cuando, en 1924, fue a Copenhague, invitado por Bohr. Llegó en el momento de máximas dificultades para la teoría cuántica. Heisenberg consideraba que aquel período de cercana colaboración con Bohr había sido infinitamente instructivo para él, a pesar de que fue el período en que las dificultades y las contradicciones internas en teoría cuántica se habían hecho más desconcertantes. Fue una época dura porque los físicos cuánticos se enfrentaban a problemas conceptualmente muy abstrusos pero fue también fructífera, puesto que le ofreció la oportunidad de contribuir de forma prominente a la formulación de la nueva mecánica cuántica ${ }^{3}$ que surgió de aquella profunda crisis. Así, entre 1925 y 1927, Heisenberg publicó 11 artículos científicos originales, la mayoría de los cuales desempeñaron un papel crucial en la creación y la interpretación ${ }^{4}$ de la mecánica cuántica; con ellos precipitó la culminación de la revolución cuántica, iniciada con el siglo XX.

Heisenberg, que provenía de Gotinga, donde trabajaba con Born, se benefició de dos tradiciones y estilos científicos, diferentes pero, a la vez, complementarios entre sí. "El énfasis en Gotinga estaba más en el lado matemático, en el lado formal [...] mientras que en Copenhague estaba más en el lado, yo diría, filosófico”, decía Heisenberg (Sánchez Ron, 2001: 425). Así, para Born el conocimiento de la ciencia física había de ser, ante todo y para siempre, una descripción matemática. Sin embargo, para Bohr el cono-

\footnotetext{
${ }^{3}$ Nombre con el que Born bautizó la nueva teoría en 1924.

${ }^{4}$ La contribución de Heisenberg constituye un componente fundamental de la interpretación de Copenhague.
} 
cimiento en física había de provenir, en primer lugar, de una comprensión intuitiva, que era resultado de una "intensa ocupación” en los fenómenos físicos estudiados; y solo posteriormente esta comprensión directa se había de estructurar a través de un "completo análisis racional", en forma matemática. Así, Heisenberg escribía: "Noté que la claridad matemática no tenía en sí misma valor para Bohr. Temía que la estructura matemática formal oscureciera el núcleo físico del problema y, en cada caso, estaba convencido de que una completa explicación física había de preceder a la formulación matemática" (Heisenberg, 1967: 98). Esta podía ser una de las razones por las que Heisenberg pensaba que "Bohr era principalmente un filósofo, no un físico" (Heisenberg, 1967: 95). En cambio, Heisenberg se sentía fascinado por la sencillez y la claridad de la abstracción matemática, que ejercía "una mágica atracción" sobre él y le llevaba a pensar que en el formalismo matemático estaba la clave que permitiría rescatar la teoría cuántica de la crisis en la que estaba sumergida.

\subsection{El nacimiento de la mecánica de matrices}

En setiembre de 1925 Heisenberg publicó un artículo de título modesto, "Sobre una reinterpretación teórico-cuántica de las relaciones cinemáticas y mecánicas”, pero de ambicioso objetivo:"establecer una base para la mecánica cuántica teórica basada exclusivamente en relaciones entre cantidades que en principio sean observables" (Heisenberg, 1967: 261).

Heisenberg empezó su artículo argumentando que los problemas que presentaba la teoría cuántica justificaban la necesidad de realizar profundas innovaciones en ella. Había varias razones para ello. En primer lugar, las 
reglas que se usaban en teoría cuántica para calcular magnitudes observables, como la energía del átomo de hidrógeno, contenían relaciones entre cantidades que, en principio, eran inobservables, como la posición y el período de revolución del electrón. En realidad solo se podían observar las fotografías de Wilson ${ }^{5}$, los resultados del experimento que se describía explicando que los rayos $\beta$ emitidos por elementos radioactivos "causaban" ${ }^{6}$ la condensación de pequeñas gotas cuando atravesaban vapor de agua supersaturado y estas gotitas dejaban huellas que formaban líneas "irregularmente curvas" (Heisenberg, 1930: 4). En segundo lugar, las reglas que se usaban para calcular magnitudes observables solo se podían aplicar al átomo de hidrógeno, no a otros átomos con varios electrones. Por tanto, estas reglas, que se derivaban de la mecánica clásica, no habían de aceptarse en la cuántica — con excepción de la condición de frecuencia de Einstein-Bohr, $\mathrm{E}_{\mathrm{mn}}=b v_{n}-b v_{m}$, que siempre funcionaba- Así pues, no podía mantenerse la validez de la mecánica clásica para solventar los problemas de la teoría cuántica. Por estas razones, los físicos teóricos, tanto de la escuela de Gotinga como de Copenhague, esta-

${ }^{5}$ Fotografías tomadas en la cámara de niebla, que es el aparato, diseñado por C.Wilson, que contiene como medio detector un vapor supersaturado que se condensa en pequeñas gotas líquidas alrededor de los iones producidos por el paso de partículas cargadas, como las partículas $\alpha$ o $\beta$.

${ }^{6}$ A pesar de que, como veremos, Heisenberg reiteradamente negó la relación de causalidad nunca dejó de utilizar la noción de causa. Se justificaba alegando que el físico cuántico necesita el lenguaje común para comunicar sus descubrimientos aunque este lenguaje sea anterior a la física atómica y por esta razón no contenga en sus términos las consecuencias de los descubrimientos realizados en el dominio atómico (Heisenberg, 1966: 47). 
ban buscando una nueva mecánica, que Born ya había llamado "mecánica cuántica". Heisenberg, en la introducción de su artículo, describía la situación con estas palabras:

En esta situación parece sensato abandonar toda esperanza de observar, hasta este momento, cantidades inobservables, tales como la posición y el período del electrón y conceder que el acuerdo parcial de las reglas cuánticas con la experiencia es más o menos fortuito. En su lugar me parece más razonable intentar establecer una mecánica cuántica teórica, análoga a la mecánica clásica, pero en la cual solo haya relaciones entre cantidades observables (Heisenberg, 1967: 262).

A través de estas palabras podemos intuir la manera de trabajar de la mayoría de los físicos cuánticos del momento. Como hemos mencionado anteriormente, su metodología consistía básicamente en adaptar las fórmulas clásicas al lenguaje de la teoría cuántica, siguiendo la guía del principio de correspondencia (Bohr, 1988: 88). Así, el procedimiento utilizado para resolver cada problema era diferente y se necesitaba grandes dosis de destreza e intuición para "adivinar" la solución. Heisenberg, por el contrario, buscaba el esquema formal de una nueva teoría que, absorbiendo en sus fundamentos el principio de correspondencia, permitiera solucionar los problemas de una manera matemáticamente justificada y rigurosa (Jammer, 1989: 211).

La "reinterpretación” de Heisenberg comenzaba considerando la radiación emitida por un electrón que se mueve. En teoría cuántica no es posible, según Heisenberg (Heisenberg, 1967: 262), asignar al electrón una posición espacial por medio de magnitudes observables. Pero, en cambio, sí es posible observar la intensidad de la radiación que emite el electrón. Y Heisenberg 
reemplazó las órbitas por una serie ordenada de entidades matemáticas que representaban la radiación. Estas entidades fueron posteriormente identificadas como los elementos de un objeto matemático que entonces era poco conocido, la matriz.

En el proceso de la creación de la mecánica de matrices se pueden distinguir tres pasos (Cassidy, 1992: 197): En primer lugar, Heisenberg reinterpretó las ecuaciones de la cinemática clásica del espacio y el tiempo como fórmulas no clásicas en mecánica cuántica, utilizando las propiedades observables de la radiación emitida por osciladores virtuales atómicos. En segundo lugar, Heisenberg impuso el criterio positivista de la observabilidad de todas las cantidades como un postulado básico de la teoría. Y, en tercer lugar, sustituyó las órbitas mecánicas por los elementos de la matriz.

Pero las matrices no disfrutan de la propiedad conmutativa. Y el hecho de haber de renunciar a la conmutatividad fue interpretado por Heisenberg como el precio que era necesario pagar para obtener el formalismo matemático adecuado para la descripción de los estados atómicos. Este proceso matemático recuerda el principio epistemológico general que, ocho años después, Heisenberg expresaba con las palabras siguientes:

Casi cada progreso en ciencia ha sido pagado con un sacrificio, porque casi cada nuevo logro intelectual ha tenido que abandonar posiciones y concepciones previas. De esta manera, el incremento de conocimiento y de profundización disminuye continuamente la pretensión del científico de "comprender" la naturaleza (Heisenberg, 1985: 29).

Así, el artículo de Heisenberg de 1925 inició la mecánica de matrices. Y, dos meses más tarde, Born y Jordan, continuando el trabajo de Heisenberg, 
publicaron un nuevo artículo en el que convertían el cálculo de matrices de Heisenberg en una "teoría sistemática de mecánica cuántica" (van der Waerden, 1967: 277), que constituyó la primera formulación rigurosa de la mecánica de matrices. En él se reinterpretaba casi cada variable y función como la posición y el momento- de la mecánica clásica como una matriz cuántica.

En 1926, Born, Jordan y Heisenberg publicaron un nuevo artículo (van der Waerden, 1967: 321) con el que continuaban los dos artículos anteriores, el de Born y Jordan y el de Heisenberg. Este artículo fue de fundamental importancia para la historia de la mecánica cuántica, porque ofrecía una exposición lógicamente consistente de la mecánica de matrices, que constituía la primera exposición completa de los fundamentos de la mecánica cuántica. Era un sistema matemático de gran simplicidad, construido lo más paralelamente posible, según los autores, a la teoría clásica, de tal manera que podía considerarse como una formulación del principio de correspondencia de Bohr, del principio heurístico que había actuado como guía metodológica de Bohr y Heisenberg.

\section{Las relaciones de indeterminación}

\subsection{Circunstancias en las que fueron desarrolladas}

Las relaciones de indeterminación constituyen, junto al postulado cuántico, al principio de complementariedad de Bohr (Bohr, 1988: 99) y a la interpretación estadística de Born, el núcleo esencial de la interpretación de 
Copenhague. Estas relaciones fueron derivadas en 1927 por Heisenberg ${ }^{7}$ en Copenhague en las siguientes circunstancias. En 1926 Schrödinger había dado, en Munich, un seminario sobre su nueva mecánica de ondas al que había asistido Heisenberg, que presentó (Jammer, 1974: 56) unas objeciones que no fueron comprendidas por muchos de los asistentes, entre los cuales se encontraba W.Wien, quien, al responder a las palabras de Heisenberg, había señalado que Schrödinger había probado de una vez por todas que los "saltos cuánticos" eran absurdos y así había puesto fin a una teoría basada en nociones $\tan$ confusas como esta. Inmediatamente después, Heisenberg había escrito a Bohr sobre "el desafortunado desenlace de la discusión" (Heisenberg, 1967: 100) y fue probablemente el contenido de dicha carta el que provocó que Bohr invitara a Schrödinger a pasar unos días en Copenhague, para tratar de la interpretación de la mecánica cuántica. Schrödinger aceptó. Naturalmente, Heisenberg también viajó a Copenhague; "no quería perderme estas discusiones tan importantes" (Heisenberg, 1967: 101), declaró años después.

Aunque Schrödinger había demostrado la equivalencia formal entre su mecánica de ondas y la mecánica de matrices, había una gran distancia entre las interpretaciones conceptuales subyacentes a las dos formulaciones. En primer lugar, por una parte, la significación física de la mecánica de matrices quedaba oscurecida por lo abstracto que, en la época, resultaba el manejo de las matrices. En cambio, la mecánica de ondas utilizaba el aparato familiar de

${ }^{7}$ Aunque Dirac ya se le había anticipado en el trabajo que publicó en 1926 (Jammer, 1974: 61). 
las ecuaciones diferenciales propias de la mecánica de fluidos, que sugería una representación fácilmente visualizable. Por otra parte, la mecánica de matrices rehuía cualquier representación intuitiva y pictórica y renunciaba a una descripción clásica en el espacio y el tiempo.

Pero la diferencia más significativa entre la interpretación de Heisenberg y la de Schrödinger era la concepción del átomo. La de Heisenberg acentuaba el elemento de discontinuidad, puesto que representaba los electrones como bolas de materia cargada que giraban y las transiciones entre estados estacionarios de energía del átomo como saltos cuánticos. En cambio, la de Schrödinger acentuaba el elemento de continuidad, puesto que representaba las partículas por paquetes de ondas cuyas trayectorias permitían inferir las trayectorias macroscópicas observadas de las partículas e interpretaba los estados estacionarios como modos armónicos de vibración de las ondas y los saltos cuánticos como transiciones continuas de un modo de vibración a otro (Schrödinger, 1982: 11).

Fue durante la visita de Schrödinger a Copenhague cuando el conflicto entre los dos puntos de vista se hizo más abierto y profundo. La atmósfera que se respiraba en las discusiones se muestra claramente en la descripción que ofrece Heisenberg:

Pese a que Bohr solía ser muy respetuoso y amable al tratar con las personas, en esta ocasión parecía casi un fanático inflexible que no estaba dispuesto a transigir frente a su interlocutor ni permitirle a este la más mínima vaguedad. Es imposible reproducir la pasión con la que ambas partes discutían; tampoco puedo describir lo profundamente ancladas que estaban las convicciones que se atisbaban tras las palabras de cada uno... [En 
El viraje epistemológico de Heisenberg

aquellas conversaciones] cada uno luchaba con todas sus fuerzas por la interpretación de la recién obtenida representación matemática de la naturaleza (Heisenberg, 1967: 101).

Esta carga emocional que se daba en el enfrentamiento entre las dos concepciones se percibe también en las famosas palabras que Schrödinger exclamó: "Si realmente existen todos estos malditos saltos cuánticos he de lamentar haberme dedicado a la teoría cuántica”, a lo cual Bohr respondió:"Pero nosotros le estamos muy agradecidos por lo que hizo, ya que su trabajo ha hecho avanzar mucho la teoría" (Jammer, 1974: 57).

Aunque cada uno se mantuvo en su posición, el debate entre Schrödinger y Bohr evidenció la necesidad de clarificar la relación entre la teoría y los datos empíricos. Heisenberg, que participó activamente en las discusiones que se dieron en el Instituto Bohr, veía que se disponía de una descripción matemática libre de contradicciones pero (Heisenberg, 1967: 105) no se sabía cómo usarla para la descripción de situaciones experimentales tan simples, como, por ejemplo, la traza de un electrón en una cámara de niebla. Dirac y Jordan habían desarrollado la teoría matemática, basada en el trabajo de Born y Jordan, y a Heisenberg y a Bohr les parecía que, si bien la compleción de este formalismo matemático les confirmaba su convicción de que nada se había de cambiar en la estructura formal de la mecánica cuántica, quedaba por solventar el gran problema de la interpretación de esta estructura, de "expresar la conexión, sin ninguna contradicción, entre dicha estructura y el experimento" (Heisenberg, 1967: 105). Así pues, cuando Schrödinger partió, Heisenberg y Bohr se quedaron en Copenhague tratando de encontrar una interpretación general de la mecánica cuántica y analizando para ello "experimentos mentalmente posibles" (Heisenberg, 1967: 104). 
Ni Bohr ni Heisenberg sabían cómo interpretar el sencillo experimento del paso del electrón tal como aparecía en la cámara de Wilson, puesto que no veían cómo podían unir de forma coherente, por una parte, el formalismo matemático de la mecánica de matrices, en el cual la trayectoria de un electrón no estaba definida porque no era observable y, por otra parte, las huellas del electrón observadas en la cámara de niebla. Cuando Bohr abandonó Copenhague para unas cortas vacaciones, Heisenberg siguió, a solas, reflexionando sobre este problema y, una noche, recordó la conversación que había mantenido con Einstein en la primavera de 1926. Cuando Heisenberg le había mostrado su opinión —en la más pura línea positivista - de que una buena teoría física solo había de operar con cantidades observables, Einstein había respondido: "Solo la teoría decide qué es lo que se puede observar" (Heisenberg, 1967: 106). Entonces Heisenberg pensó que esta respuesta era la clave de la solución del problema y salió a pasear por el parque que hay junto al Instituto, meditando sobre las consecuencias de las palabras de Einstein. Pensó que decir, como habitualmente se decía, que se observaba la trayectoria del electrón en la cámara de niebla era hablar demasiado a la ligera; quizás solo se pudiera decir que se observaba "una sucesión discreta de puntos del electrón imprecisamente determinados"; porque realmente "de hecho, solamente se ven unas pocas gotitas de agua aisladas en la cámara, seguramente mucho más extensas que un electrón”. Por tanto, se había planteado mal la pregunta de cómo, en la mecánica cuántica, se podía representar matemáticamente la trayectoria del electrón en la cámara de niebla y la pregunta se había de plantear así: "¿Se puede representar, en la mecánica cuántica, una situación en la que un electrón se encuentre aproximadamente 
—es decir, con una cierta imprecisión — en un lugar dado y además posea aproximadamente — también con cierta imprecisión — una velocidad dada?" (Heisenberg, 1967: 106).

Heisenberg regresó al Instituto, hizo los cálculos, vio que dicha situación sí se podía representar matemáticamente y calculó que el producto de las imprecisiones no podía ser menor que un número positivo que cumplía una relación con el quantum de Planck. Así concluyó que "quedaba finalmente establecida la conexión entre las observaciones en la cámara de niebla y la matemática de la mecánica cuántica" (Heisenberg, 1967: 106).

\subsection{Derivación de las relaciones de indeterminación}

Buscando la conexión entre las observaciones en la cámara de niebla y el formalismo matemático y utilizando la teoría de Dirac-Jordan, Heisenberg obtuvo las relaciones de indeterminación, representadas de la siguiente forma:

$$
\delta p \delta q=h / 2 \pi
$$

...según la cual el producto de la indeterminación en el momento y la indeterminación en la posición es constante y, por consiguiente, nunca puede ser nulo.

Así, Heisenberg probó que, según el formalismo matemático de la mecánica cuántica, no se podían conocer exactamente - es decir, sin indeterminación - y simultáneamente la posición y la velocidad de una partícula. Le faltaba probar si esta conclusión era compatible con la máxima precisión obtenible en los experimentos de medida. Pensaba que sí, "porque los mismos procesos que tienen lugar durante el experimento, durante la 
observación, deben acatar las leyes de la mecánica cuántica” (Heisenberg, 1967: 107). Y se dispuso a estudiar la cuestión.

Partió del principio operacional según el cual un concepto científico constituye un código condensado de operaciones y su significado es una relación definida de impresiones sensoriales del observador. Así, Heisenberg escribió en su histórico artículo de 1927:

Cuando se quiere clarificar qué se entiende por las palabras "posición del objeto", por ejemplo, del electrón (relativa a un determinado sistema de referencia), entonces se debe especificar definidos experimentos con cuya ayuda se pueda medir "la posición del electrón"; si no es así esta palabra no tiene significado (Heisenberg, 1983: 64).

Aquí Heisenberg (Heisenberg, 1967: 107) se ayudó del recuerdo de una conversación que había mantenido con B. Drude, un compañero de estudios de Gotinga, cuando debatían sobre las dificultades de la representación de las trayectorias electrónicas en el átomo. Drude había sugerido construir un microscopio de tan gran poder de resolución que permitiera ver directamente la trayectoria de un electrón. Tal microscopio no podría funcionar con luz visible, pero sí con rayos $\gamma$, porque, según las leyes ópticas de resolución, la precisión del microscopio es mayor cuanto menor es la longitud de onda $\lambda$ de la radiación que ilumina ${ }^{8}$. De esta manera, Heisenberg pensó en el experi-

\footnotetext{
${ }^{8}$ Según la siguiente relación, que ofrece el límite de exactitud en la posición: $\Delta x=\lambda \sin \alpha$ (en la cual $\Delta x$ es la mínima distancia a la que se pueden distinguir objetos, $\lambda$ es la longitud de onda de la luz utilizada y $\alpha$ es el ángulo a través del cual la luz choca con la partícula que se pretende observar).
} 
mento mental de intentar determinar la posición de un electrón con la ayuda de un microscopio de rayos $\gamma$; pero entonces se encontró con el efecto Compton, es decir, con el hecho de que

...en el instante en el que se determina la posición — por tanto, en el momento en el que el fotón choca con el electrón - el electrón sufre un cambio discontinuo en el momento. Este cambio es mayor cuanto menor es la longitud de onda de la luz utilizada, es decir, cuanto más exacta es la determinación de la posición [...] Así, cuanto más precisamente se determina la posición, menos precisamente se conoce el momento y a la inversa (Heisenberg, 1983: 64).

Así pues, el cambio discontinuo en el momento, producido por el efecto Compton, aumenta al aumentar la precisión en la localización del electrón. Y Heisenberg llegó a la conclusión de que los conceptos utilizados en teoría clásica para la descripción de un sistema mecánico podían también definirse para procesos atómicos, pero los experimentos que ofrecían tales definiciones sufrían una indeterminación introducida por los procedimientos usados para la determinación simultánea de las dos cantidades, debido a las perturbaciones generadas por los instrumentos de medida sobre los sistemas medidos, que eran provocadas por las interacciones entre los dispositivos y los objetos.

\subsection{El problema de la falta de Anschaulichkeit}

El artículo de 1927, en el que Heisenberg derivó sus relaciones de indeterminación, lleva por título "Über den anschaulichen Inbalt der quantenheoretischen Kinematik und Mechanik", traducido como "El contenido intuitivo de la mecánica y cinemática cuántica”. El término original alemán 
correspondiente a "intuitivo" es anschaulich, adjetivo que aparecía reiteradamente a lo largo del artículo, denotando claridad y visualización. La traducción más literal de anschaulich es "visualizable". Y puesto que "ver" se utiliza frecuentemente como sinónimo de "entender" o "comprender", anschaulich se puede traducir como "inteligible" o "intuitivo".

Heisenberg era consciente de que la mecánica de matrices que había creado no era anschaulich, clara y visualmente satisfactoria, como lo era la física clásica. En cambio, la mecánica de ondas de Schrödinger era considerada intuitiva y pictórica, porque retornaba a la continuidad y a la representabilidad de los fenómenos físicos en el espacio y el tiempo (Schrödinger, 1996: 489). Schrödinger había acusado a la mecánica de matrices de ser unanschaulich ${ }^{9}$ y Heisenberg, para quitarse este estigma, en su artículo de 1927 propuso modificar el significado del requerimiento de Anschaulichkeit rompiendo con la tradición clásica de la representabilidad espacio-temporal. Los electrones no eran anschaulich en sentido habitual, puesto que no podían describirse usando descripciones pictóricas cotidianas como la posición, la velocidad y la órbita. Heisenberg redefinió el término anschaulich y le otorgó el significado de satisfactorio en sentido positivista, es decir, consistente con el sistema

\footnotetext{
${ }^{9} \mathrm{El}$ ambiente intelectual alemán del momento valoraba el carácter pictórico e intuitivo, anschaulich, de las explicaciones científicas y los físicos, quizás inconscientemente, deseaban adaptarse al Zeitgeist, a la ideología dominante (Forman, 1984: 75). La frecuencia con la que aparece el término anschaulich en los trabajos de los físicos cuánticos de la época muestra el gran esfuerzo que estos estaban realizando para dotar de un significado claro al formalismo cuántico.
} 
formal y predecible sin ambigüedad a partir del resultado de los experimentos.

Así, Heisenberg pensaba que sus relaciones de indeterminación estaban tan firmemente construidas sobre la base del formalismo y de los experimentos cuánticos que las calificaba de anschaulich, es decir, claras e intuitivas, y a ello añadía afirmaciones contundentes. Así, consideraba que el formalismo de Dirac-Jordan era completo y, por tanto, las relaciones de indeterminación eran irrefutables; en consecuencia, futuros experimentos nunca alterarían la validez de los principios de la mecánica cuántica ni permitirían superar los límites impuestos por las relaciones de indeterminación ${ }^{10}$.

\section{La relación de causalidad}

La perspectiva bajo la cual Heisenberg contempló la relación de causalidad fue uno de los elementos claves de la interpretación de la mecánica cuántica que la mayoría de los físicos aceptaron. Pero vamos a ver que esta perspectiva experimentó una evolución. Así, encontramos que Heisenberg concluía su artículo de 1927 con la siguiente declaración anticausal:

Lo que es erróneo en la formulación fuerte de la ley de la causalidad, "cuando conocemos el presente exactamente, podemos predecir el futuro", no es la conclusión sino la hipótesis.

${ }^{10}$ Aunque Heisenberg no llegó a probar estas aserciones, las fue reiterando y llegaron a formar parte de la interpretación de Copenhague. 
En principio no podemos conocer el presente con todo detalle. Por esa razón cada cosa observada es una selección de un conjunto de posibilidades y una limitación sobre lo que es posible en el futuro. Como el carácter estadístico está tan íntimamente relacionado con la inexactitud de todas las percepciones es posible preguntar si todavía hay oculto, tras el universo estadístico de la percepción, un mundo 'verdadero', en el que la ley de la causalidad sería válida. Pero tal especulación nos parece, por decirlo explícitamente, inútil y sin sentido. La Física solo debe describir la correlación de las observaciones. Uno puede expresar el verdadero estado de los hechos mejor de esta manera. Ya que todos los experimentos están sujetos a las leyes de la mecánica cuántica y, por tanto, de la relación anterior $\left(p_{1} q_{1} \approx h\right)$ se sigue que la incorrección de la ley de la causalidad es una consecuencia definitivamente establecida de la mecánica cuántica (Wheeler y Zurek, 1983: 84).

Este pasaje tuvo gran repercusión ${ }^{11}$. En primer lugar, muestra la conclusión filosófica que el mismo Heisenberg extrajo de sus relaciones de indeterminación. Esta conclusión ofrece una indeterminación por imprecisión y una indeterminación acausal, puesto que la indeterminabilidad de los valores iniciales impide la predictibilidad de los valores futuros y niega un significado operacional a la causalidad.

En el enunciado condicional que constituye la formulación fuerte ${ }^{12}$ de la ley de la causalidad, "si conocemos con precisión el presente, entonces somos

${ }^{11}$ En los meses siguientes a la publicación del artículo hubo numerosas manifestaciones de físicos alemanes que declaraban que la mecánica cuántica mostraba que la ley de causalidad no se podía mantener.

${ }^{12}$ Heisenberg se estaba refiriendo a la noción laplaciana de determinismo, que consideraba equivalente a la noción kantiana de causalidad. No podía aceptar dicha noción en física atómica, porque en este dominio se presentan situaciones como la siguiente: cuando un átomo emite partículas no puede predecirse el tiempo exacto en que tendrá lugar la emisión, tan solo 
capaces de predecir el futuro" el antecedente nunca puede ser verdadero; por tanto, infirió Heisenberg, el enunciado condicional es falso. Esta solución que ofreció Heisenberg al problema de la causalidad resultó sorprendente en la filosofía moderna, porque, a pesar de que el problema que con ella pretendió solventar había sido debatido durante muchas generaciones, nunca se le había dado esta respuesta.

Sin embargo, la refutación que Heisenberg dio a la ley de causalidad no es lógicamente correcta, porque un enunciado condicional no es falso cuando lo es el antecedente. Por esta razón, Bergmann (Jammer, 1974: 74), en 1929, mantenía que la mecánica cuántica no refutaba la ley de la causalidad, aunque sí mostraba su inaplicabilidad. Pero, desde el punto de vista operacionalista de Heisenberg en 1927, “inaplicabilidad” e "invalidez” eran sinónimos.

Este carácter positivista aparece también en la anterior cita de Heisenberg en la que hemos visto que manifestaba que no tiene sentido la pregunta de si, en el fondo, existe también un mundo real causal, oculto tras el mundo fenoménico, aparentemente no causal. No tiene sentido porque la ciencia física se ha de limitar a describir relaciones entre observaciones. Mas dicha manifestación de positivismo no era consistente con el punto de partida del argumento que había llevado a Heisenberg a las relaciones de indeterminación, con el enunciado de Einstein de que "solo la teoría decide qué es lo que puede observarse”. Y con el tiempo Heisenberg cambió su posición respecto al positivismo.

el tiempo promedio; así pues, no podemos encontrar un hecho anterior al cual deba seguir, según una regla, la emisión (Heisenberg, 1959: 68). 
Y también cambió su actitud respecto a la relación de causalidad. Así, en unas conferencias impartidas tres años después, en 1930, mostró dicho cambio. Siguió formulando la ley de la causalidad con el siguiente enunciado: "Si en un cierto momento se conocen todos los datos de un sistema dado entonces es posible predecir de forma inambigua el comportamiento físico del sistema también en el futuro" (Heisenberg, 1984: 45); pero manifestó que en este enunciado condicional lo que es falso es el consecuente - recordemos que anteriormente había incidido en el hecho de que era falso el antecedente-, aunque el antecedente sea verdadero, porque el acto de la medición cuántica tan solo puede ofrecer resultados probabilísticos. Así, en Diciembre de 1930, en Viena, manifestaba que: "El segundo miembro de la formulación clásica de la causalidad es falso, porque a partir de la función de Schrödinger el contenido físico de los sistemas en general solo puede predecirse estadísticamente“(Heisenberg, 1984: 45). Y unos meses antes, en Königsberg, había ilustrado esta idea con la siguiente comparación:

\footnotetext{
Mientras que en la teoría clásica el estado del sistema se caracterizaba por la información de las coordenadas y el impulso, en la teoría cuántica se representa por una Función en el espacio de configuraciones, que indica cuán probable es que las coordenadas y los impulsos tengan determinado valor, si los medimos. Esta función de estado ("Función de Schrödinger") permite así en general solo afirmaciones inexactas sobre el futuro de un experimento (Heisenberg, 1984: 44).
}

No obstante, sorprendentemente, Heisenberg en la misma conferencia ofrecida en Viena había admitido que hay casos especiales en los que-"también se puede mantener un cierto 'grado de determinismo' — si puedo 
decirlo- en la teoría atómica” (Heisenberg, 1984: 37). Así, una causalidad restringida se podía mantener con pleno significado. Y continuaba: "Si en un cierto momento se conocen todos los datos de un sistema aislado, existen, en cualquier momento posterior, experimentos el resultado de los cuales puede ser exactamente predicho, si el sistema no está sujeto a otras perturbaciones que las necesarias para la realización del experimento". Y, reconociendo el asombro que sus manifestaciones podían provocar, añadía: ”Esta formulación es algo incómoda, pero muestra claramente los límites dentro de los que se puede mantener la mecánica cuántica como teoría causal. Si exactamente indica una ley de la causalidad o no es naturalmente una pura cuestión de gusto”.

Por todo ello no sorprende que, años después, en 1952 encontremos las siguientes manifestaciones de Heisenberg en las que negaba la relación de causalidad con suma cautela:

Una de las más interesantes consecuencias generales de la moderna Física atómica la constituyen las transformaciones que bajo su influjo ha sufrido el concepto de las leyes naturales o de la regularidad de la Naturaleza. En los últimos años, se ha hablado a menudo de que la moderna Física atómica parece abolir la ley de la causa y el efecto o por lo menos dejar parcialmente en suspenso su validez ${ }^{13}$ (Heisenberg, 1984: 376).

En síntesis, como hemos podido apreciar, hubo una evolución en la actitud de Heisenberg ante la ley de la causalidad. En primer lugar, había negado la causalidad; por dos razones: porque no se pueden conocer todas las

${ }^{13} \mathrm{E} 1$ subrayado es nuestro. 
condiciones iniciales y porque la función de onda solo ofrece resultados probabilísticos; pero posteriormente la había llegado a admitir, en el caso de que fuera posible conocer todas las condiciones iniciales de un sistema cerrado en el que se pudieran controlar todas las perturbaciones provocadas por el experimento. Mas hay que precisar que dicho control solo es alcanzable en ciertos tipos de experimentos. Por ejemplo, si en un sistema cerrado se ha medido una determinada magnitud dinámica del sistema y, por consiguiente, el sistema se encuentra en un estado propio de dicha magnitud, una nueva medida de la misma magnitud dará un resultado determinado, pero no una medida de una magnitud dinámica complementaria, puesto que en este último caso las perturbaciones son incontrolables.

\section{Actitud de Heisenberg ante el positivismo}

\subsection{La inspiración positivista}

En un principio, especialmente en sus históricos artículos de 1925 y 1927, vemos un Heisenberg positivista y operacionalista. Así Heisenberg, como positivista, mantenía que la física había de limitarse a la descripción de las relaciones entre percepciones, que la trayectoria del electrón existía solo cuando la observábamos, que cuando no se observaba el electrón no podía decirse nada de él y, por consiguiente, no podía tampoco saberse qué hacía ni qué era. Por otra parte, como operacionalista, mantenía que un concepto físico solo tenía significado si se indicaba un procedimiento definido para medir la cantidad referida por dicho concepto. 
Sommerfeld, profesor y mentor de Heisenberg, consideraba-(Jammer, 1974: 58) que este era un devoto seguidor de Mach. Además a Heisenberg, marcado por el positivismo de principios de la década de los 20, Wittgenstein le había impresionado vivamente (Jammer, 1989: 209). Así, percibimos que en los primeros trabajos de Heisenberg resuenan las últimas palabras del Tractatus, "De lo que no se puede hablar, hay que guardar silencio" (Wittgenstein, 1981: 152).

Heisenberg, al buscar el significado de los enunciados que utilizaba, se encontraba con el hecho de que términos fundamentales de la vida cotidiana y de la física clásica no tenían sentido en la teoría cuántica. Así, repetía con frecuencia (Jammer, 1989: 210) que la estrategia de Einstein al empezar a construir su teoría de la relatividad constituía, para él, un modelo a imitar. De la misma manera que este había visto que no tenía sentido hablar de la simultaneidad de dos hechos espacialmente distantes antes de realizar una adecuada sincronización de los relojes, así también Heisenberg consideró que no tenía sentido hablar de la posición de una partícula con una velocidad definida antes de describir un experimento por el cual dicha posición pudiera medirse.

La inspiración positivista que la teoría especial de la relatividad generó en Heisenberg y le ayudó a crear su mecánica de matrices es recogida por el mismo Heisenberg en este fragmento:

En esas circunstancias recordé una idea que había leído en algún libro de Einstein: una teoría física solo debe manejar magnitudes que puedan observarse directamente. Este requisito garantizaba [...] el nexo entre las fórmulas matemáticas y los fenómenos. Al hilo de esa idea se llegaba a un formalismo matemático que realmente parecía cuadrar con los 
fenómenos atómicos. En colaboración con Born, Jordan y Dirac fue luego elaborado en una mecánica cuántica cerrada de aspecto tan convincente que en verdad no cabía ya ninguna duda. Pero todavía no sabíamos cómo interpretar esa mecánica cuántica, cómo hablar de su contenido-(Heisenberg, 1980: 121)

Así pues, vemos en esta cita que Heisenberg estaba muy seguro del formalismo de la nueva mecánica cuántica, pero no estaba tan seguro de su interpretación, de su significado físico.

En sus dos artículos fundamentales, el de 1925 y el de 1927, Heisenberg propuso basar la teoría cuántica "exclusivamente en relaciones entre cantidades que en principio fueran observables" (Heisenberg, 1967: 261), entre magnitudes que se las pudiera medir a través de un procedimiento bien definido. El significado de muchos términos de la vida cotidiana y de la física clásica provenía de procedimientos que no se podían aplicar a la teoría cuántica. Así, por ejemplo, la distancia entre objetos constituía una noción creada por los primeros geómetras que se podía definir colocando una regla de medida entre ellos y contando cuantas veces podía ponerse - extremo a extremo- entre el primer y el segundo objeto. Generalizando esta experiencia fue posible definir una relación de esta naturaleza en el mundo macroscópico. Y sobre ella se formaron diferentes conceptos: longitud, posición, espacio, velocidad, trayectoria..., que constituyeron las coordenadas mentales de nuestro pensamiento.

Estas coordenadas mentales, formadas para los fenómenos más grandes, que, por ser inmediatamente perceptibles a nuestros sentidos, constituyen nuestra experiencia común, están profundamente enraizadas en nuestros hábitos de pensamiento y "embalsamadas en nuestro lenguaje" (Lindemann, 
1932: 15$)^{14}$; pero estas coordenadas mentales no son adecuadas para los fenómenos más pequeños, que solo son perceptibles a través de unos procedimientos experimentales que no permiten medir la distancia con precisión; el antiguo concepto de distancia y las nociones correlativas que se convirtieron en parte importante de nuestro subconsciente mental no han de referirse a las partículas subatómicas, puesto que estas solo se pueden medir usando otras partículas que, al interferir con ellas, cambian las magnitudes que se pretenden medir. Mas nuestro lenguaje está construido con estos términos habituales y nos es muy difícil pensar en otros distintos. De ahí surge el gran problema que representa el lenguaje en teoría cuántica. El lenguaje habitual fue construido para describir un conjunto de experiencias mucho más pobre del que hemos adquirido con los nuevos descubrimientos científicos.

Así, hemos visto que Heisenberg en su artículo de 1925 rechazó nociones utilizadas en la teoría cuántica anterior, como la posición, la órbita y el período de revolución del electrón en el átomo, porque al ser inobservables, "les faltaba una evidente fundamentación física" (Heisenberg, 1967: 261) y las reemplazó por nociones como las frecuencias de oscilación y las amplitudes, que determinan la intensidad de las líneas. "Al menos estas magnitudes sí podían ser observadas de forma directa” (Heisenberg, 1967: 85) ${ }^{15}$. Y hemos visto también que en su artículo de 1927 concluía que especular sobre la

\footnotetext{
${ }^{14}$ Con este libro Lindemann pretendía mostrar que el misterio que rodeaba a la mecánica cuántica y las dificultades que esta teoría presentaba podían clarificarse con la adecuada comprensión de las relaciones de indeterminaión de Heisenberg.

${ }^{15}$ Pero no se podía observar la órbita de los electrones en los experimentos.
} 
cuestión de si tras el mundo probabilístico que se percibe hay un mundo regido por la ley de la causalidad no tiene sentido y es inútil, ya que la física solo ha de describir relaciones entre observaciones.

\subsection{La influencia de la crítica de Einstein}

Pero estas manifestaciones positivistas contrastan con el punto de partida del razonamiento que, como hemos visto, llevó a las relaciones de indeterminación, el enunciado de Einstein de que solo la teoría determina lo que puede observarse. Relata Heisenberg que este enunciado inspiró el trabajo que le llevó a derivar las relaciones de indeterminación. Dicho enunciado le llevó a cambiar su pregunta inicial de ¿Cómo representar la órbita del electrón en la cámara de niebla, donde vemos el electrón moviéndose a lo largo de una trayectoria? por esta otra: ¿No es verdad que siempre (en plena Naturaleza y en la cámara de niebla) ocurren solo situaciones que pueden ser descritas en el formalismo matemático de la mecánica cuántica? (Heisenberg, 1984: 433).Y utilizando el formalismo de Dirac y Jordan, Heisenberg llegó a la conclusión de que no se podían describir al mismo tiempo la posición exacta y la velocidad de un electrón, es decir, llegó a las relaciones de indeterminación.

Sin embargo, en el mismo artículo de 1927 Heisenberg-empezaba a mostrar un cambio en su perspectiva epistemológica y comenzaba a alejarse del positivismo — siguiendo la enseñanza de Einstein de que solo la teoría determina lo que se puede observar-, aunque aún mantenía elementos positivistas. Posteriormente abandonó el positivismo y llegó incluso a adoptar una posición crítica con esta corriente de pensamiento. Era la actitud 
crítica cuyas semillas Einstein había sembrado en la conversación que en 1926 había impresionado vivamente a Heisenberg y poco a poco había ido creciendo en la mente de este (Heisenberg, 1980: 122). Dicho diálogo había sido iniciado por Einstein preguntando por la filosofía que subyacía a la nueva teoría - la mecánica cuántica- que Heisenberg había creado. Este le respondió manifestando que la característica más destacada de dicha teoría era la de "introducir solo cantidades observables" (Heisenberg, 1984: 428), que realmente pudieran ser observadas, siguiendo el ejemplo de Einstein, quien al crear la teoría de la relatividad especial había abandonado la noción de tiempo absoluto.

En la misma conversación Einstein había reprochado a Heisenberg que en su descripción matemática de la mecánica cuántica no aparecieran las órbitas de los electrones, puesto que le parecía absurdo "querer suprimir por completo las órbitas“(Heisenberg, 1967: 88). Según Einstein, si existían las trayectorias en el macrocosmos también habían de existir en el microcosmos, puesto que la noción de trayectoria no podía depender del tamaño del cuerpo. Heisenberg se defendió de esta crítica siguiendo un criterio positivista y aduciendo que las órbitas de los electrones no se podían observar y lo único que se podía registrar realmente eran las frecuencias y las intensidades de la luz emitida por los átomos. Así vemos que Heisenberg, contrariamente a Einstein, presuponía diferentes nociones para el macrocosmos y para el microcosmos. Si lo más prudente y riguroso era introducir solo magnitudes directamente observables, el concepto de órbita del electrón no podía aparecer en la teoría (Heisenberg, 1967: 92). 
Pero Einstein respondió que cualquier teoría entrañaba magnitudes inobservables y el principio positivista que estaba defendiendo Heisenberg de solo admitir magnitudes observables no se podía llevar de forma consecuente a la práctica (Heisenberg, 1980: 122). Heisenberg le replicó que él simplemente estaba utilizando la misma filosofía positivista que Einstein había utilizado en su teoría de la relatividad, al excluir nociones como el espacio y el tiempo absolutos porque no podían observarse y al usar una definición operacional de simultaneidad, a lo cual Einstein respondió:

Quizás he usado este tipo de filosofía, pero de todos modos es absurda. Dicho de manera más cauta, pienso que tal vez sea heurísticamente valioso acordarse de lo que se observa en realidad. Sin embargo, desde el punto de vista de los principios, es un error el querer basar una teoría exclusivamente en las magnitudes observables, pues en la realidad sucede justamente lo contrario. Solo la teoría decide qué es lo que se puede observar. Verá usted, la observación es, en general, un proceso muy complejo (Heisenberg, 1967: 89).

Veamos otros fragmentos que muestran la profunda impresión que los argumentos de Einstein ejercieron en Heisenberg:

(Einstein) Me hizo notar que incluso el concepto de observación era de suyo problemático. Toda observación — argumentaba — presupone que entre el fenómeno a observar y la percepción sensorial que finalmente entra en nuestra conciencia existe una relación unívoca y conocida. Pero de esa relación solo podríamos estar seguros si conociésemos las leyes de la naturaleza que la determinan. Ahora bien, cuando es preciso poner en duda esas leyes - como sería el caso de la moderna física atómica-, entonces el concepto de "observación” pierde también su claro significado. Entonces es la teoría la que determina lo que puede observarse. Tales consideraciones me eran completamente nuevas y ejercieron sobre mí una honda impresión; desempeñaron también más tarde un papel importante 
El viraje epistemológico de Heisenberg

en mis trabajos y se revelaron harto fructíferas en el desarrollo de la nueva física (Heisenberg, 1980: 123).

Veamos ahora cómo Heisenberg expresaba algunas de estas consideraciones:

Si puedes observar una cosa o no depende de la teoría que uses. Es la teoría la que decide qué puede observarse. La observación significa que establecemos una conexión entre un fenómeno y nuestra conciencia del fenómeno [...] Einstein me había señalado que es realmente peligroso decir que solo se ha de hablar de cantidades observables. Porque cada teoría razonable, junto a todas las cosas que se pueden observar inmediatamente, también ofrece la posibilidad de observar otras cosas más indirectamente [...] Cuando tienes la mecánica cuántica tú no puedes solo observar frecuencias y amplitudes, sino, por ejemplo, amplitudes de probabilidad, ondas de probabilidad, etc. y estos, desde luego, son objetos completamente diferentes (Heisenberg, 1984: 429).

Hay que tener en cuenta (Sánchez Ron, 2001: 438) que este Einstein que dialogaba con Heisenberg en 1926 no era el que, en 1905, había defendido, al desarrollar la teoría especial de la relatividad, que la física solo había de manejar observables, como Mach había propuesto (Mach, 1987: 43). Mach había reconocido los puntos débiles de la mecánica clásica y uno de estos puntos débiles era la noción newtoniana de espacio absoluto. Así, la crítica efectuada por Mach a dicha noción había significado para Einstein el punto de partida de su teoría de la relatividad, puesto que, según Mach, el espacio absoluto constituye un "concepto patológico", ya que es "una pura construcción mental que no se puede encontrar en la experiencia" (Holton, 1982: 167). Y, bajo la influencia del principio de economía de pensamiento, Eins- 
tein había rechazado la existencia del espacio y tiempo absolutos porque no eran observables (Einstein, 1949: 53).

El Einstein que había inspirado el principio básico de Heisenberg de considerar solo magnitudes observables para crear su formalismo de mecánica de matrices era el Einstein que, en su juventud, había visto la necesidad de crear la teoría especial de la relatividad por las contradicciones en las que caía la física clásica al intentar interpretar de forma consistente determinados experimentos ${ }^{16}$. Entonces, "la ciencia fue forzada a admitir que uno de los supuestos de esta interpretación clásica no estaba basado en ninguna observación directa ya que se refería a dominios inaccesibles a la observación directa" ${ }^{17}$ (Heisenberg, 1966: 12). La retórica de Heisenberg recordaba la de Einstein en 1905 y sonaba como la exhortación de los positivistas: "Limpia la física extrayendo las reminiscencias de metafísica, religión y misticismo" (Heelan, 1975: 125).

Pero la observabilidad a que se refería Heisenberg no era la simple observabilidad directa. Los observables que Heisenberg mencionaba no eran magnitudes observables simple e inmediatamente a través de los sentidos. Eran magnitudes que aunque no se pudieran percibir directamente sí se podían medir — como las magnitudes propias del dominio cuántico-. Así, lo

\footnotetext{
${ }^{16}$ Especialmente el de Michelson.

${ }^{17}$ Como el supuesto de que, sin haber sincronizado los relojes, dos hechos espacialmente distantes pueden ser simultáneos. Así, Heisenberg valoraba especialmente que Einstein hubiera excluido nociones que no se podían observar, como el espacio y el tiempo absolutos, y que hubiera utilizado una definición operacional de la simultaneidad de dos hechos (Cassidy, 1992 :239).
} 
que movía a Heisenberg era el hecho de que ciertas variables que aparecían en la antigua teoría cuántica, como las órbitas del electrón en el interior del átomo, no eran medibles. Así, aunque los niveles de energía, los electrones intraatómicos, el spin y otras entidades tampoco eran observables directamente, se distinguían de las órbitas de los electrones dentro de los átomos en el hecho de que tenían efectos medibles. Para Heisenberg una magnitud observable significaba que se podía medir y el principio de observabilidad consistía en aceptar solo enunciados que tuvieran consecuencias observables. Cualquier cosa que no tuviera "consecuencias observacionales", que no fuera "experimentalmente verificado", era excluido del dominio de la ciencia física (Heisenberg, 1930: 1).

El Einstein que en 1926 mantuvo la célebre conversación con Heisenberg era el que, diez años antes, había ampliado la teoría especial de la relatividad a la teoría general en la que intentaba relacionar los fenómenos de la gravitación con las relaciones de masa en el mundo cuatridimensional. Así, escribió Einstein: "Mi alejamiento del positivismo se dio solo cuando trabajaba en la teoría general de la relatividad" (Fine, 1986: 86). Para desarrollar esta teoría, Einstein se había distanciado del pensamiento de Mach, puesto que este le resultaba excesivamente restrictivo; el requisito positivista de Mach constituía una simplificación excesiva para una teoría cuyos enunciados generales estaban muy alejados del dominio de la directa observación empírica. Para Mach los principios fundamentales de una teoría habían de contener conceptos que fueran muy cercanos a la observación inmediata y el camino que asciende desde esta hasta aquellos había de ser breve y fácilmente inteligible. En cambio, para Einstein era largo y difícil (Einstein, 1934: 163). El ideal de Mach 
era la cauta síntesis de hechos observacionales que pueden ser llevados inmediatamente a la llana y cruda percepción sensorial (Mach, 1948: 28). Pero la teoría general de la relatividad contiene el concepto de campo, que está lejos de ser directamente observable. Así pues, comparando la teoría especial y la teoría general de la relatividad, Heisenberg manifestaba años después:

\footnotetext{
La teoría general de la relatividad añade a la revisión del concepto de tiempo una revisión de las propiedades geométricas del espacio. Si la teoría está interpretando correctamente el pequeño número de observaciones astronómicas que ahora poseemos entonces, como es bien conocido, ha de existir una relación entre la geometría y la distribución de la materia en el universo. La teoría general de la relatividad ya no se apoya en una base experimental segura como la teoría especial, aunque ningún experimento la ha contradicho hasta el presente. Su poder convincente no se basa en la interpretación de muchos resultados experimentales, que no podemos evaluar en el presente, sino en un nuevo método de pensamiento-(Heisenberg, 1966: 14).
}

Aunque al joven Heisenberg, en el diálogo que mantuvo con Einstein en 1926, le sorprendió la actitud que este mostró, sus argumentos le parecieron evidentes (Heisenberg, 1967: 90). Años después, manifestó que, aunque lo que había considerado más valioso de su filosofía era haber utilizado solo cantidades observables, Einstein le había corregido "esta opinión" (Heisenberg, 1984: 428). Así, encontramos la actitud crítica que, ante el positivismo, mantenía Einstein ${ }^{18}$ en su madurez también en Heisenberg. Este, con el paso del tiempo, se fue sintiendo cada vez más insatisfecho con el positivismo (Heelan, 1965: 137), fue comprendiendo que la actividad científica contenía

${ }^{18}$ En qué sentido entendía la observabilidad nunca fue exactamente formulado por Einstein, pero su visión sobre la observabilidad cambió radicalmente durante el período entre la relatividad especial (1905) y la general (1915) (Heelan, 1975: 125). 
elementos básicos de universalidad y necesidad que el positivismo no contemplaba y fue desarrollando una concepción propia de la ciencia en la que aparecieran dichos elementos, sugeridos en gran parte por la naturaleza de sus nuevas investigaciones en el dominio de las partículas elementales y los rayos cósmicos.

Así, en paralelo a su distanciamiento del positivismo, encontramos en Heisenberg su dedicación a un trabajo de investigación en un dominio alejado de la directa observabilidad empírica que exige el positivismo, un trabajo dirigido a la búsqueda de una teoría del campo unificado, que uniera los campos conocidos, partiendo del estudio de los rayos cósmicos y de las colisiones de partículas producidas en los nuevos aceleradores ${ }^{19}$.

Al igual que Einstein (Einstein, 1949: 684), Heisenberg también mantenía que había que adaptar la propia filosofía a la experiencia. Por consiguiente, cuando los nuevos fenómenos que se perciben presentan un carácter diferente al de los que se han percibido anteriormente, han de cambiar los esquemas mentales que ayudan a entenderlos. Así pues, ante nuevos descubrimientos empíricos hay que modificar el marco filosófico en que situarlos. En palabras de Maxwell (Pais, 1988: 454), "la ciencia experimental nos está continuamente revelando nuevos hechos de los procesos naturales y

\footnotetext{
${ }^{19}$ Entonces la física de partículas ya había entrado en la era de las grandes máquinas, como los grandes detectores y los grandes aceleradores.
} 
somos así obligados a buscar nuevas formas de pensamiento apropiadas a estos hechos" ${ }^{20}$.

Concluyendo, en síntesis, la actitud de Heisenberg ante el positivismo sufrió la misma metamorfosis que la de Einstein. En su juventud ambos fueron profundamente influidos por el pensamiento positivista, que les inspiró las creaciones más significativas de sus carreras; en cambio, posteriormente se alejaron de dicha corriente filosófica. Y este cambio radical de posición, en los dos autores, se corresponde con la evolución que ambos realizaron, en su trabajo de investigación, hacia temas más amplios y abstractos, a los cuales dedicaron grandes esfuerzos durante el resto de sus vidas. Así pues, Einstein en su madurez se entregó especialmente a la construcción de la teoría general de la relatividad y Heisenberg se volcó en la teoría de campo unificado. Pero previamente Heisenberg había intentado encontrar la matriz-S, la matriz de scattering, el operador que recogiera la evolución de un sistema de partículas elementales durante su colisión a grandes velocidades.

\subsection{Nuevos campos de investigación}

\section{La matriz-S}

En sus primeros intentos por construir la matriz-S Heisenberg aún mantenía un espíritu positivista, que consideraba que le había sido muy productivo en los exitosos trabajos de su juventud, los cuales habían culmina-

${ }^{20}$ Podemos ver esta actitud como acorde a la actitud general que mantiene la persona que calificamos de racional e inteligente, cuyo pensamiento se adapta continuamente a la siempre cambiante experiencia cotidiana. 
do en los artículos de 1925 y de $1927^{21}$, en los que había desarrollado su mecánica de matrices y sus relaciones de indeterminación. Respecto a los inicios de su búsqueda, había manifestado lo siguiente: "Recordé la vieja historia de los observables [...] y pensé que sería útil preguntar '¿qué podemos realmente observar en una colisión entre partículas elementales?' y fue natural mantener que la matriz-S o matriz de scattering era una base racional para una teoría” (Heisenberg, 1984: 435). Así, el título de su primer artículo dedicado a la matriz-S, publicado en 1943, fue "Las 'cantidades observables' en la teoría de partículas elementales", en el que Heisenberg intentó, como había hecho con éxito en sus trabajos de los años 20, utilizar solo variables observables y excluir de su teoría todas las inobservables.

En su búsqueda de la matriz-S propuso la hipótesis de la existencia de una longitud mínima fundamental, $l \approx 10^{-13} \mathrm{~cm}$, para limitar los posibles cambios en el momento y la energía de dos partículas que chocaran a elevada velocidad. La longitud mínima fundamental constituía una limitación que permitía identificar observables que eran medibles en experimentos de laboratorio. Heisenberg consideraba que los hechos acaecidos a un lado de dicho límite eran observables y los acaecidos al otro lado eran inobservables.

Para estudiar el choque de dos partículas, Heisenberg trabajaba con los observables constituidos por las propiedades de las partículas consideradas mucho antes y mucho después de la colisión-(Cassidy, 1992:477). En el pasado remoto estaban en estado de partículas libres, que llamamos $n$. Mucho después de la colisión, las nuevas partículas que se habían producido se su-

\footnotetext{
${ }^{21}$ que le habían situado entre los físicos dirigentes del siglo XX.
} 
ponía que se encontraban prácticamente también en estado libre, que llamamos $m$. Heisenberg representaba las partículas como ondas planas y la colisión como una matriz de transformación ( 2 por 2 ) de las ondas de antes a las ondas de después de la colisión. Esta matriz ofrecía la probabilidad de transición del estado $n$ al $m$. Heisenberg seguía una estrategia similar a la que había seguido en 1925 y le había llevado al descubrimiento de la mecánica de matrices pero en esta nueva ocasión no le llevó a conseguir su objetivo, ya que la matriz-S resultó ser "poco más que una vasija que contenía datos experimentales bien ordenados" (Pais, 1988: 498); según Pauli, solo era un concepto vacío (Cassidy, 1992: 498). Las dificultades con las que se encontró Heisenberg en su búsqueda de la matriz $\mathrm{S}$ propiciaron que su interés se dirigiera a la investigación de un nuevo dominio, la física del campo cuántico, dominio en el cual propuso su teoría de campo unificado.

\section{La teoría de campo unificado}

Supongamos que en un momento, que según la mecánica cuántica no se puede predecir, un átomo excitado experimenta una transición a su estado fundamental mientras emite un fotón. Nos podemos preguntar: ¿Dónde estaba anteriormente el fotón? La observación empírica nos da la respuesta: En ningún sitio. Por esta razón concluimos: Ha sido creado en el acto de la transición. En otro momento, que tampoco se puede predecir, un núcleo beta-radioactivo se convierte en otro núcleo diferente mientras emite un electrón y un neutrino. ¿Dónde estaba antes? En ningún lugar. Por consiguiente, podemos pensar que ha sido creado en el proceso de la desintegración beta. En otro momento un átomo absorbe un fotón y entra en un estado excitado. ¿Dónde está después de la absorción? En ningún lugar. 
También podemos pensar que ha sido aniquilado en el mismo momento de la absorción.

El marco teórico que describe cómo las partículas y la radiación son creadas y destruidas es la teoría del campo cuántico, teoría construida con el objetivo de comprender la estructura de la materia y la descripción de los procesos más elementales de la Naturaleza. Dicha teoría ofrece una técnica para calcular las probabilidades de creación, de aniquilación y de colisión de toda clase de partículas - fotones, electrones, positrones, mesones,...- a través de métodos de sucesiva aproximación ${ }^{22}$.

En esta teoría los postulados de la relatividad especial (debido a las elevadas velocidades a que se mueven las partículas que chocan) y los de la mecánica cuántica llegan a una síntesis ${ }^{23}$ sin sufrir ninguna alteración. La teoría del campo cuántico tuvo unos espléndidos inicios - a finales de los años 20 y principios de los 30 - pero después entró en una fase caracterizada por la confusión, las dudas y la incertidumbre que sentían los físicos que a ella se dedicaban, puesto que comprobaban que las predicciones teóricas no se adaptaban adecuadamente a las observaciones empíricas, ya que solo coincidían en una primera aproximación.

${ }^{22}$ No se ha obtenido una rigurosa expresión que permita calcular la probabilidad de estos procesos (Pais, 1988: 325).

${ }^{23}$ En cambio, en los albores de la mecánica cuántica - hasta finales del año 1926- la teoría especial de la relatividad y la teoría cuántica habían jugado roles separados en el estudio de las partículas y los campos. 
Heisenberg, con gran coraje, participó —como Fermi, Pauli,...- en el ambicioso proyecto de construir esta teoría, que estaba basada en la noción de un campo portador de las fuerzas entre partículas. Las partículas y sus interacciones eran representadas por campos que satisfacían las ecuaciones diferenciales relativistamente invariantes. Previas teorías habían tratado partículas y campos como entidades distintas. En cambio, la nueva teoría unificaba estas entidades y las trataba como un solo campo general de materia, que Heisenberg simbolizaba como $\psi(x)$. Entonces las distintas partículas elementales aparecían como estados estacionarios de energía de este campo general. Para este campo general de materia Heisenberg presentó en 1947 una sencilla ecuación diferencial que contenía el campo $\psi(x)$ y la longitud mínima fundamental $l$. Con esta ecuación Heisenberg determinaría las masas de las partículas elementales condensadas a partir del campo de materia. Y las reglas de selección y las leyes de conservación de las propiedades de las partículas emergerían de las propiedades de simetría de la ecuación de campo.

Heisenberg había construido una teoría verdaderamente unificadora porque toda la materia y sus interacciones podían reducirse a una simple ecuación para un campo unificado que representaba cada forma de materia y cada tipo de fuerza. Constituía una noción revolucionaria. Pero en 1956 T.D.Lee y C.N.Yang descubrieron que en la decadencia $\beta$ no se conservaba la paridad, es decir, se violaba la simetría bajo la reflexión espacial. Heisenberg escribió una nueva ecuación, que presentó en 1958. Pero tampoco consiguió su objetivo. Y en la última fase de su vida se dedicó, por una parte, 
a continuar trabajando en su teoría de campo unificado ${ }^{24} \mathrm{y}$, por otra, a situar su trabajo en una perspectiva filosófica, que derivaba directamente de la antigua filosofía griega.

\subsection{La noción de partíaula elemental}

¿Podemos concebir mentalmente que dividimos un pedazo de materia, lo volvemos a dividir una y otra vez y así infinitamente? ¿Podemos imaginarlo? ¿Podemos hacerlo realmente? ¿Encontraremos al final unas últimas partículas elementales que sean indivisibles? Estas preguntas nos las planteamos actualmente, como también se las formularon los primeros filósofos griegos, y han sido abordadas una y otra vez a lo largo de la historia del pensamiento. Las respuestas que se han ofrecido a través del tiempo han seguido los caminos que ellos trazaron. Por esta razón, a pesar de los siglos transcurridos, Heisenberg valora la aportación de los primeros pensadores de occidente; sin embargo, muestra también que la física atómica contemporánea ha ofrecido una respuesta nueva.

Según Heisenberg, los griegos rechazaron la divisibilidad infinita de la materia porque tenían problemas con la noción de continuidad, como muestran las paradojas de Zenón de Elea. Por esta razón, Leucipo y Demócrito ofrecieron su hipótesis de la existencia de los átomos, partículas indivisibles e invariables que constituían la materia. Heisenberg interpreta esta hipótesis como una solución al eterno problema de comprender la multiplicidad de los

${ }^{24}$ No se ha realizado aún una adecuada evaluación histórica y científica de la aportación de Heisenberg en esta etapa de la historia de la física (Cassidy, 1992:543). 
seres a partir de una única entidad fundamental, el átomo. La forma, el orden y la posición de los átomos determinan las diferencias entre unos seres y otros y sus movimientos mecánicos determinan el cambio de los seres en el mundo. Si bien la hipótesis atómica da un paso adelante en la dirección correcta, porque, según Heisenberg, reduce las múltiples propiedades observaobservadas (color, olor, sabor) en la materia a la forma y situación de los átomos, la solución materialista y mecanicista de Leucipo y Demócrito no le satisface, puesto que no ofrece una ley general que determine la posición y el movimiento de los átomos, una ley que permita explicar la estructura de la materia.

Por esta razón, Heisenberg prefiere la solución idealista que Platón presenta en Timeo 54b, concepción que considera opuesta a la de Leucipo y Demócrito (Heisenberg, 1969: 28). Según Platón, la materia está formada de cuerpos regulares (por ejemplo, cubos, tetraedros y octaedros) que se componen de triángulos. Así, las partículas más pequeñas del elemento tierra se equipararían al cubo y las del fuego al tetraedro. Por tanto, los cuerpos regulares son cuerpos simétricos, que se caracterizan por sus propiedades de simetría en relación a la transformación de rotación en el espacio. Obviamente, los triángulos de los que se componen los cuerpos regulares, no son materia sino forma matemática.

La solución atomista se adoptó como la más adecuada durante los siglos en que crecieron las ciencias naturales pero posteriormente los físicos observaron que el átomo no era indivisible sino que estaba compuesto de protones, electrones y neutrones. Y después descubrieron las llamadas "partículas elementales", de corta vida, como los mesones. Mas, ante dichos 
descubrimientos, reaparece la pregunta inicial: ¿Se pueden dividir las partículas elementales? Experimentalmente la división solo se puede realizar disparando a gran velocidad unas partículas elementales sobre otras. Y aquella pregunta solo se puede responder observando si dichas partículas se descomponen formando unidades más pequeñas.

Esto se ha realizado en los aceleradores de partículas y en ellos las partículas elementales producidas no son más pequeñas que aquellas a las que se hizo chocar. Por consiguiente, en el choque de partículas elementales, estas no se descomponen. Lo que hay es una producción de materia por energía. Según la teoría de la relatividad, de la energía puede surgir materia y la materia puede transformarse en energía; por tanto "materia y energía son equivalentes”. La energía cinética de las partículas que chocan a gran velocidad produce nuevas partículas. Así pues, la conclusión que se infiere es que se ha de renunciar a la noción de partícula elemental como unidad fundamental más pequeña (Heisenberg, 1969:30). Esta es la nueva respuesta que ha dado la naturaleza al antiguo dilema de qué ocurre cuando se sigue dividiendo la materia.

Así, la física de las partículas elementales daba la razón a Platón porque "los últimos componentes de la materia son figuras matemáticas caracterizadas por sus propiedades de simetría" (Heisenberg, 1969: 31). El rasgo más destacado de la ecuación universal de materia de Heisenberg son sus simetrías. Por esta razón, su perspectiva filosófica se basa en las relaciones de simetría, la esencia de dicha ecuación. Así pues, la estructura del espacio y el tiempo se expresa por la invarianza de todas las ecuaciones bajo el grupo inhomogéneo de Lorentz. Los invariantes de este grupo son la masa y el 
spin. Otras simetrías de campo deducen otras propiedades que caracterizan las partículas elementales, como la carga eléctrica, el número bariónico y leptónico y la extrañeza.

Por esta razón, Heisenberg encuentra en Platón la expresión de su idea motriz: la estructura básica subyacente de los fenómenos no viene dada por entidades materiales sino por la forma matemática que determina la estructura de estas entidades, en la cual se muestra la simplicidad de los fenómenos y la unidad subyacente al mundo. Las formas matemáticas — como las simetrías - simplifican y ordenan el mundo fenoménico, que aparece a simple vista como desordenado, confuso y caótico. En definitiva, las formas matemáticas nos ayudan a comprender el mundo.

Mas ¿qué es comprender? Ante esta cuestión Heisenberg responde que: “Comprender es ordenar los fenómenos en torno a un principio unitario. La única manera de lograrlo es captando las semejanzas y regularidades de los fenómenos [...] Comprender la cambiante multiplicidad de los fenómenos es una búsqueda de ese principio básico subyacente" (Heisenberg, 1974: 171). Es decir, "comprender significa reconocer interconexiones y ver lo particular como caso especial de lo más general" (Heisenberg, 1974: 211). Las matemáticas constituyen la forma de expresar nuestra comprensión de la naturaleza. Muy joven (Heisenberg, 1966: 36), al leer el Timeo de Platón, había sido impresionado por la noción de que tras el mundo de los sentidos hay un mundo real de puras formas matemáticas, que nos permite comprender el primero. Y precisamente la teoría de la relatividad y la teoría cuántica son más abstractas que las teorías físicas anteriores porque se alejan mucho más que estas de la observación directa (Heisenberg, 1974: 212) que prescri- 
be el positivismo. Por esta razón, a Heisenberg, en su madurez, le parecía que la perspectiva positivista no ayudaba a comprender el mundo.

\subsection{Posición crítica ante el positivismo}

La visión que del positivismo tenía Heisenberg en su madurez se muestra (Heisenberg, 1967: 265) en una conversación que mantuvo con W.Pauli en 1952, después de una conferencia que se realizó en Copenhague para restablecer las relaciones entre científicos de todo el mundo y tratar de la construcción de un gran acelerador en Europa. A la conferencia asistieron muchos positivistas, que no mostraron ninguna oposición a la mecánica cuántica, en contra de lo que esperaban Heisenberg y Pauli. Según este último, ello fue debido al hecho de que los positivistas veían que la mecánica cuántica describía correctamente los fenómenos cuánticos y para los positivistas no había nada más allá de los fenómenos, pues mantenían que "el mundo es todo lo que sucede" y "el mundo es la totalidad de los hechos, no de las cosas" (Wittgenstein, 1981: 67). Mas los positivistas no aceptaban nociones añadidas por los físicos a los hechos cuánticos, nociones como la complementariedad y las relaciones de indeterminación que consideraban que constituían un "complemento lírico poco claro, un retroceso al pensamiento precientífico, en resumen, pura palabrería" (Heisenberg, 1967: 266).

Para los positivistas, según Heisenberg, comprender equivalía a poder pronosticar. En cambio, para este había una gran diferencia entre ambos conceptos y, con el fin de explicarla, ofreció la siguiente imagen: 
Cuando vemos un avión en el cielo podemos calcular con relativa certeza dónde estará un segundo después. Tan solo hay que seguir la trayectoria en línea recta; si advertimos que el avión traza una curva, podemos incluir esta curvatura en nuestro cálculo. Lo más probable es que acertemos en la mayor parte de los casos. Pero eso no quiere decir que hayamos comprendido la trayectoria, pues esto solo es posible si hablamos previamente con el piloto y este nos informa sobre el vuelo que va a realizar (Heisenberg, 1967: 266).

Cuando Pauli preguntó a Heisenberg qué quería decir con esta comparación y qué se correspondía en la ciencia de la naturaleza con la intención del piloto, este le respondió con otra comparación: la astronomía de Ptolomeo y la teoría del movimiento de los planetas de Newton. Según el criterio de verdad del pronóstico, la $1^{\mathrm{a}}$ no es inferior a la $2^{\mathrm{a}}$, pero a todos nos parece más correcta la $2^{\mathrm{a}}$ que la $1^{\mathrm{a}}$, porque, según Heisenberg, Newton "describió la intención según la cual está construida la naturaleza”. Las leyes fundamentales son elementos significativos del plan según el cual está ordenada la naturaleza y se puede plantear la cuestión de si detrás de dichas leyes hay una conciencia, cuya intención revelan estas leyes. Dirían los positivistas, según Heisenberg, que esta pregunta no ha de plantearse, porque no tiene un significado claro. Para los positivistas hay que dividir el mundo entre lo que se puede decir con claridad y lo que se debe callar. "Por tanto aquí tendríamos que callarnos. Pero no hay filosofía tan carente de sentido como esta, porque no hay casi nada que se pueda expresar claramente. Si se elimina todo lo oscuro, probablemente solo nos quedarían algunas tautologías absolutamente faltas de interés" (Heisenberg, 1967: 275). Si el ser humano se limitara a estudiar lo que es sencillo, se dejaría muchos temas interesantes para estudiar, precisamente aquellos de los cuales los positivistas prohíben hablar. Así 
pues, en síntesis, se puede decir que Heisenberg aceptaba de los positivistas sus exigencias de precisión y claridad, pero no sus restricciones y prohibiciones.

El concepto de verdad de Heisenberg era "completamente diferente al de los positivistas" que se derivaba de "la posibilidad de pronosticar" (Heisenberg, 1967: 273). Encontrar la verdad según Heisenberg era descubrir el plan con el cual la naturaleza está construida, como la intención con la cual el piloto realiza su vuelo. Con esta imagen antropomórfica pretendía representar las leyes de la naturaleza y la interconexión que hay entre ellas, que en mecánica cuántica se expresan mediante el lenguaje formal de las matemáticas.

Las matemáticas ofrecen orden y simplicidad, dos de los perennes valores de la ciencia, puesto que los científicos consideran "lo ordenado como lo bueno y lo confuso como lo malo" (Heisenberg, 1967: 276) y buscan establecer un orden unitario sobre campos muy amplios, orden que expresan mediante fórmulas generales. Este orden, este plan, que siguen los procesos concretos que ocurren en la naturaleza, actúa como una brújula que nos orienta para comprender el mundo de la experiencia.

Para comprender el mundo, el ser humano, a lo largo de toda su historia, ha buscado encontrar los grandes nexos causales que relacionan los hechos concretos. Durante siglos no se preocupó de avalar el descubrimiento de estos nexos, de estas interconexiones generales, con la experiencia. Como consecuencia de ello, según Heisenberg, "se extendió toda clase de supersticiones" en nombre de la ciencia. Pero en el siglo XVII la ciencia se dirigió a 
la experiencia y con su cambio de actitud y de método inició un camino firme y seguro.

Los positivistas, según Heisenberg, intentan justificar el procedimiento de la ciencia moderna con un sistema filosófico que prohíbe la reflexión sobre las cuestiones más generales, porque su contenido no puede percibirse directa e inmediatamente a través de los sentidos y ello les priva de claridad conceptual. Pero siguiendo escrupulosamente tal prohibición no se podrían comprender teorías como la mecánica cuántica. La física, según el Heisenberg maduro, no consiste solo en experimentos y formulismos matemáticos, puesto que necesita una interpretación filosófica que enlace ambos ámbitos y así ayude a entender el mundo que percibimos. Heisenberg no podía aceptar que se renunciara a la búsqueda de esta interpretación filosófica por el hecho de que dicha interpretación no pudiera expresarse con absoluta claridad.

Cuando los positivistas manifestaban que sobre lo que no se puede hablar con claridad hay que callar, Heisenberg respondía que esta "es una filosofía sin sentido, porque no hay casi nada que se pueda expresar claramente. Si se eliminara todo lo oscuro probablemente solo nos quedarían algunas tautologías, absolutamente desprovistas de interés" (Heisenberg, 1967: 273). Las limitaciones impuestas por los positivistas llevarían a abandonar el principal objetivo de la investigación científica, la comprensión de los grandes nexos generales que unen los hechos concretos y particulares que percibimos en el mundo. Nos obligarían a quedarnos en la detallada y precisa descripción de estos. Nada más. Por ello Heisenberg no podía aceptar que se renunciara a 
comprender el plan con el que le parecía que estaba trazado el universo, el plan que lo hacía inteligible ${ }^{25}$.

Sin embargo, en 1955-Heisenberg (Heisenberg, 1959) manifestaba su aprecio por el espíritu crítico del positivismo moderno y consideraba justificado su postulado general de que si un determinado enunciado tiene sentido siempre existe la posibilidad de que sea analizado detallada y críticamente, rememorando la proposición del Tractatus: "Lo que se puede decir se puede decir claramente" (Wittgenstein, 1981: 65). A través de la historia la sospecha de que un enunciado podía carecer de significado había sido muy útil porque había abierto la puerta a la posibilidad de nuevos conceptos ${ }^{26}$.

Así, según Heisenberg, la tesis positivista de que todo conocimiento está, en último término, basado en la experiencia había llevado a la exigencia de la clarificación lógica de cualquier enunciado de las ciencias naturales. No obstante, esta exigencia no se había podido satisfacer en la teoría cuántica, puesto que términos como "posición" y "velocidad" de un electrón, por ejemplo, no estaban claramente definidos en dicha teoría. Ello nos muestra que nunca podremos saber por anticipado cuáles son las limitaciones que se nos presentarán para aplicar nuestros conceptos cuando realicemos nuevos descubrimientos y ampliemos nuestros conocimientos. Y Heisenberg llegaba a

${ }^{25}$ Así, el mismo Heisenberg se calificaba de platónico y pitagórico (Heisenberg, 1969: 28; 1966: 35) (Heelan, 1965: 139), puesto que, como Einstein (Einstein, 1949: 684), estaba convencido de que la tarea del físico consiste esencialmente en la búsqueda de las estructuras abstractas que, en el fondo, rigen el mundo que percibimos.

${ }^{26}$ Por ejemplo, la pregunta ¿en qué órbita se mueven los electrones alrededor del núcleo? 
declarar: "Por consiguiente, en el proceso de profundización nos vemos muchas veces obligados a emplear nuestros conceptos en una forma que no está justificada y que no tiene sentido" (Heisenberg, 1959: 74). Y, en consecuencia, insistir radicalmente en el postulado positivista de la necesidad de una clarificación lógica completa de todos los enunciados haría la ciencia imposible. Finalmente Heisenberg concluía con un antiguo adagio: "Quien nunca quiera cometer un error ha de permanecer en silencio”, sentencia que podía interpretarse como la antítesis de la última proposición del Tractatus. Así, mientras el famoso enunciado de Wittgenstein invitaba a la cautela y a la parquedad, este viejo proverbio evocado por Heisenberg invitaba a la audacia y a la creación.

\section{Condusión}

Aunque, como hemos visto, Heisenberg había manifestado que su motivación heurística era filosófica — porque declaraba que ante todo quería entender los hechos que percibía-, la actividad a la que fundamentalmente él dedicó sus esfuerzos no fue el desarrollo de sus concepciones filosóficas, sino el desarrollo formal de la fundamentación de la mecánica cuántica, campo en el cual realizó una significativa aportación, con su descubrimiento de la mecánica de matrices y de las relaciones de indeterminación, y, posteriormente, con su investigación en la teoría de partículas y en la teoría del campo unificado. Fue con la aparición de la interpretación de Copenhague, nacida (Cassidy, 1992: 255) con el artículo de Heisenberg de 1927, que empezaron 
las sistemáticas manifestaciones filosóficas a través de las cuales Heisenberg divulgaba las revolucionarias conclusiones de la nueva mecánica cuántica ${ }^{27}$.

Hemos visto que Heisenberg no mantuvo posturas unitarias durante toda su vida. Así, por ejemplo, hemos visto que empezó negando la causalidad pero posteriormente llegó a aceptar una causalidad restringida, lo cual le acercaba a la postura defendida por Einstein. Su actitud ante el pensamiento positivista experimentó una metamorfosis paralela a la que previamente había experimentado Einstein, a pesar de que ambos autores son habitualmente situados en bandos opuestos, respecto a la interpretación de Copenhague Heisenberg fue, con Bohr, uno de los creadores de dicha interpretación y Einstein fue su crítico más célebre-. Así pues, Heisenberg empezó mostrándose como positivista, porque declaraba que solo estaba dispuesto a aceptar magnitudes observables, pero posteriormente, como Einstein, rechazó el positivismo por considerar que limitaba la creación científica. Heisenberg y Einstein, como físicos, necesitaban unas concepciones filosóficas que favorecieran el progreso de la investigación científica. Y para el progreso de esta pensaban que lo más útil era no atenerse sin reservas a ninguna directriz filosófica y, por consiguiente, mantener siempre en suspenso los principios filosóficos con la esperanza de aprender siempre de ellos.

\footnotetext{
${ }^{27}$ Dichas manifestaciones tuvieron en general muy buena acogida entre el público al que iban dirigidas, puesto que se adaptaban a las características del pensamiento dominante en la sociedad germánica de la época (Forman, 1984: 102); por ejemplo, la negación de la causalidad que realizaba Heisenberg encajaba perfectamente con la mentalidad general de la sociedad culta del momento.
} 
En conclusión, vemos que Heisenberg mostró, como Einstein, y como otros de los creadores de la mecánica cuántica — como Bohr, de Broglie o Schrödinger-, los grandes retos conceptuales que lanzaba esta teoría, las luces y las sombras de una teoría que presentaba - y presenta - grandes éxitos científicos pero también sorprendentes preguntas filosóficas y desconcertantes dilemas epistemológicos como la necesidad de limitarse a observar o atreverse a especular.

\section{Bibliografia empleada}

Bohr, N. La teoría atómica y la descripción de la Naturaleza. Madrid, Alianza Editorial, 1988.

Cassidy, D. Uncertainty. The life and science of Werner Heisenberg. New York, W.H.Freeman and Company, 1992.

Einstein, A. "On the Method of Theoretical Physics". Philosophy of Science, vol. 1, 1934.

__."Autobiographical Notes". Albert Einstein: philosopher-scientist (Ed. Paul Schilpp) Illinois, The Library of Living Philosophers, 1949, pp. 1-96.

—_. "Remarks to the Essays Appearing in this Collective Volume". Albert Einstein: philosopher-scientist (Ed. Paul Schilpp) Illinois, The Library of Living Philosophers, 1949, pp. 663689.

Fine, A. The Shaky Game. Realism and the Quantum Theory. Chicago, University of Chicago Press, 1986.

Forman, P. Cultura en Weimar, causalidad y teoria cuántica: 1918-1927. Madrid, Alianza Editorial, 1984.

Heelan, P.A. Quantum Mechanics and Objectivity. A Study of the physical philosophy of W. Heisenberg. The Hague, Martinus Nijhoff, 1965.

_—. "Heisenberg and radical theoretic change". Zeitschrift für allgemeine Wissenschafstheorie $\mathrm{VI} / 1,1975$. 
Heisenberg, W. The Physical Principles of the Quantum Theory. New York, Dover Publications, 1930.

——. "Die Rolle der Unbestimmtheitsrelationen in der modernen Physik". Monatshefte für Mathematik und Physik, 38, 365-372, 1931.

__.."Kausalgesetz und Quantenmechanic". Erkenntnis zugleich Annalen der Philosophie 2, 172-182, 1931.

—_.”Atomphysik und Kausalgesetz". Merkur 6, 701-711, 1952.

—_. Física y filosofía. Buenos Aires, La isla, 1959.

—_. Philosophic Problems of Nuclear Science. New York, Fawcett World Library, 1966.

—_. La parte y el todo. Pontevedra, Ediciones Ellago, 1967.

—_. "Problemas filosóficos de la Física de las partículas elementales". Real Academia de Ciencias Exactas, Físicas y Naturales. Tomo LXIII, cuaderno 3º 1969. Versión on line.

——. Más allá de la física. Madrid, Biblioteca de autores cristianos, 1974.

—. Encuentros y conversaciones con Einstein y otros ensayos. Madrid, Alianza Editorial, 1980.

—. Gesammelte Werke. Abteilung C, Band I. München, R. Piper\&Co. K.G., 1984.

—. Gesammelte Werke. Abteilung C, Band II. München, R. Piper\&Co. K.G., 1984.

Holton, G. Ensayos sobre el pensamiento científico en la época de Einstein. Madrid, Alianza Editorial, 1982.

Jammer, M. The Philosophy of Quantum Mechanics. New York, John Wiley \& Sons, 1974.

- The Conceptual Development of Quantum Mechanics. New York, Tomash Publishers, 1989.

Lindemann, F. El significado físico de la teoría cuántica. Oxford, Clarendon Press, 1932.

Mach, E. Conocimiento y error. Buenos Aires, Espasa-Calpe, 1948.

—_. Análisis de las sensaciones. Barcelona, Altafulla, 1987.

Messiah, A. Mecánica Cuántica. Madrid, Editorial Tecnos, 1983.

Pais, A. Inward Bound. Oxford, Clarendon Press, 1988.

Platón. Obras completas. Madrid, Aguilar, 1974.

Sánchez Ron, J.M. Historia de la física cuántica. El periodo fundacional (1860-1926). Barcelona, Crítica, 2001.

Schrödinger, E. Collected Papers on Wave Mechanics. New York, Chelsea Publishing Company, 1982. 
Josefa Castellà

La nueva mecánica ondulatoria. Heisenberg, Bohr, Schrödinger. Física cuántica. Barcelona, Biblioteca Universal del Círculo de Lectores, 1996.

Van der Waerden, B.L. (Ed). Sources of Quantum Mechanics.New York, Dover Publications, 1967.

Wheeler, J.A. y Zurek, W.H. (Ed). Quantum Theory and Measurement. Princeton, Princeton University Press, 1983.

Wittgenstein, L. Tractatus logico-philosophicus. Barcelona, Laia, 1981.

Josefa Castellà Cid

jcastellacid@hotmail.com 